\section{Trastorno por Déficit de Atención e Hiperactividad en estudiantes en edad escolar de 6 a 17 años}

\author{
Attention Deficit and Hyperactivity Disorder in school-age \\ students from 6 to 17 years old
}

\section{Martha Patricia Saldaña-Núñez* Joel Alanya-Beltran*}

\section{RESUMEN}

El presente trabajo evidencia una revisión sistemática de la literatura de diferentes artículos acerca del Trastorno por Déficit de Atención e Hiperactividad, el cual afecta a millones de niños y adolescentes en edad escolar de 6 a 17 años. Objetivo. Identificar las metodologías trabajadas en estudiantes que presentan el Trastorno de Déficit de Atención e Hiperactividad y sus niveles de relación entre pares y el rendimiento en sus clases. Materiales y métodos. El proceso de búsqueda se realizó a partir de las bases de datos: Psyclnfo, Dianet, MedLine, Redalyc, Scielo y PubMed, Revistas Neurológicas, Revistas Sicológicas, con palabras claves en inglés y español y determinados criterios de inclusión y exclusión. Resultados. Se utilizaron 18 artículos para el análisis de texto completo y 18 artículos para la revisión sistemática, los cuales evidenciaron diversos aspectos relacionados con estudiantes que presentan el Trastorno de Déficit de Atención e Hiperactividad. Conclusión. La investigación realizada podrá ser de utilidad para investigadores, docentes y profesionales del área de la salud y la educación que trabajen con niños y adolescentes con Trastorno de déficit de atención e hiperactividad.

\footnotetext{
* Licenciada en Educación con especialidad en Historia y Geografía, Maestra en Problemas de Aprendizaje, Docente, Universidad Cesar Vallejo, Lima, Perú, msaldanan@ucvvirtual.edu.pe, http://orcid.org/0000-0002-6556-446X

* Ingeniero Electrónico, Maestro en Docencia Universitaria, Docente Investigador y Temático, Universidad César Vallejo, jalanyab@ucvvirtual.edu.pe, http://orcid.org/0000-0002-8058-6229, ID Scopus: 57219413120.
}

\section{REVISTATECNOLÓGICA ciencia y educación Edwards Deming \\ ISSN: 2600-5867}

Atribución/Reconocimiento-NoCo mercial- Compartirlgual 4.0 Licencia Pública Internacional — CC

\section{BY-NC-SA 4.0}

https://creativecommons.org/licenses /by-nc- sa/4.0/legalcode.es

Editado por: Tecnológico Superior Corporativo Edwards Deming

Julio - Diciembre Vol. 5 - 2 - 2021

https://revista-edwardsdeming.com/index.php/es e-ISSN: 2576-097I

Recibido: 07 julio 2020

Aprobado: 04 Enero, 2021

Pag 61-73 
Palabras clave: Desempeño, Estudiantes, TDAH, Trastorno de déficit de atención e hiperactividad

\section{ABSTRACT}

The present work shows a systematic review of the literature of different articles about Attention Deficit and Hyperactivity Disorder, which affects millions of children and adolescents of school age from 6 to 17 years old. Objective. Identify the methodologies worked on in students with Attention Deficit and Hyperactivity Disorder and their levels of peer relationships and performance in their classes. Materials and methods. The search process was carried out from the databases: Psyclnfo, Dianet, MedLine, Redalyc, Scielo y PubMed, Neurological Journals, Psychological Journals, with keywords in English and Spanish and certain inclusion and exclusion criteria. Results. Eighteen articles were used for the full-text analysis and 18 articles for the systematic review, which revealed various aspects related to students with Attention Deficit and Hyperactivity Disorder. Conclution. The research carried out may be useful for researchers, teachers and professionals in the area of health and education who work with children and adolescents with Attention Deficit Hyperactivity Disorder.

Keywords: Performance, Students, ADHD, Attention deficit hyperactivity disorder

\section{INTRODUCCIÓN}

El Trastorno por Déficit de Atención con Hiperactividad (TDAH) es un trastorno que se presenta en el neurodesarrollo del estudiante, el cual se origina en la infancia y se mantiene durante toda la vida de la persona que lo tiene, entre las características más resaltantes tenemos: TDAH-I con predominio de inatención, TDAH-H con predominio de hiperactividad-impulsividad y TDAH-C combinado (American Psychiatric Association, 2013). En la actualidad el TDAH es considerado como uno de los trastornos del neurodesarrollo más frecuente en la población infantil (López-Martín et al, 2010), teniendo una prevalencia mundial del 5.9 a $7.1 \%$ en niños y adolescentes (Willcutt, 20I2) generando en los estudiantes dificultades en el proceso educativo ya que al presentar hiperactividad e inatención sumándose también sus problemas conductuales hacen que no puedan trabajar adecuadamente en el aula (Tigrero, 2018).

El diagnóstico del TDAH es complejo, es uno de los aspectos más estudiados, pero hasta el momento no se han encontrado evidencias de que existan marcadores neurobiológicos o neuroimágenes asociados de manera específica al TDAH (TrujilloOrrego et al, 2012). Los estudiantes con déficit de atención e hiperactividad son descritos en muchas ocasiones como niños inestables ya que la principal característica que poseen es la presencia de una gran actividad física y psíquica, en algunos casos presentan actitudes destructivas, que los llevan a tener muchas dificultades para aprender en el aula (Pineda, 2016; Chávez, 2020)

Los niños que presentan TDAH son muy hábiles, pero precisan de moverse continuamente y esto se asocia a una dificultad atencional, además, se tornan en algunos 
momentos violentos, inquietos, revoltosos, destructivos, dispersos (Pineda, 2016). Piñón et al., (2019) indican que el trastorno por déficit de atención e hiperactividad es un trastorno que generalmente aparece antes de los 12 años, iniciándose en la primera infancia teniendo un patrón persistente de conductas, falta de atención, hiperactividad, impulsividad (Silva y Velásquez, 2019)

Según American Psychiatric Association (2013) el TDAH se clasifica como un subtipo hiperactivo-impulsivo, combinado y predominio déficit atencional. Este trastorno genera que los niños y adolescentes tengan grandes dificultades para aprender y dificultades en el entorno social. El 50\% de las consultas en el área de psiquiatría infantil son de TDAH, teniendo un $10 \%$ de problemas disruptivos que suelen requerir tratamientos médicos por presentar accidentes y traumatismos debido a su gran sobre actividad física (Soutullo-Esperón y Mardomingo-Sanz, 2010). Moreno \&Martínez (2009) indican que el TDAH presenta múltiples incidencias de forma directa en el comportamiento y el aprendizaje de los estudiantes que lo padecen. Moreno y Martínez (2009) manifiestan que un niño con TDHA es difícil de manejar, porque a pesar de llevar una medicación, su conducta es extrema generando en los padres una gran carga de stress e inestabilidad emocional y en el niño una baja autoestima (Reyes, 2018), ya que no tiene una buena interrelación con sus compañeros de clases. Jiménez-Torres et al., (20l0), el rendimiento escolar es una de las variables más estudiadas en el proceso de enseñanzaaprendizaje y los factores que lo explican constituyen uno de los principales focos de interés en las investigaciones, es por eso que es muy importante observar cómo se lleva el proceso de enseñanza-aprendizaje en los estudiantes que presentan trastorno por déficit de atención con hiperactividad y su desenvolvimiento en la escuela. EI TDAH se caracteriza principalmente por presentar una gran falta de atención, exceso de actividad motora y deficiencia en el control de impulsos (Jiménez-Torres et al., 2010). La American Psychiatric Association (2013) indica que el TDAH es un trastorno del neurodesarrollo que clínicamente se define por la presencia de conductas desafiantes una gran falta de atención, mucha impulsividad y sobreactividad motora en niños, adolescentes o adultos. Los modelos que definen el TDAH se han centrado en los aspectos del funcionamiento cognitivo, atención sostenida, funciones ejecutivas que se relacionan con la memoria de trabajo, la velocidad de procesamiento y el procesamiento de la información.

Los estudios sobre los procesos de Trastorno Disocial realizados con el WISC-III en niños con TDAH han observado los beneficios de utilizar estímulos auditivos en las consignas, por lo que el test podría llegar a utilizarse como instrumento para la evaluación del TD en niños con TDAH (Yasumura et al., 2016), así mismo Gaitan \& ReyAnaconda (20I3), plantean que los lóbulos frontales realizan las funciones más avanzadas y complejas de todo el cerebro, llamadas funciones ejecutivas [FE], que son vinculadas a la intencionalidad, propósito y toma de decisiones complejas. Además, la corteza pre frontal realiza un papel fundamental en el comportamiento emocional y control de los impulsos básicos, también en memorizar secuencias de comportamiento y esquemas de acción de la memoria ejecutiva y la integración temporal de la información. Cardona \& Varela (2017) indican que el Trastorno de Aprendizaje de la Lectura presenta como 
característica dificultades en la precisión y/o fluidez en el reconocimiento de palabras, así como una baja destreza ortográfica y decodificación, que influyen en la comprensión lectora, teniendo comorbilidad entre ambos trastornos ha sido considerada fenotipo cognitivo y propuesta como uno de los modelos duales en la comprensión de la estructura cognitiva del TDAH.

Barrios et al., (2016) definen al TDAH como multifactorial y como un componente genético que puede ser heredable a un 0,76 . Además, indican que se tiene como patrón una serie de conductas persistentes con alto grado de hiperactividad e impulsividad. Por otro lado, Piñón et al., (2019), indique que la etiopatogenia del TDAH se fundamenta en una combinación de factores genéticos y ambientales, señalando que es un trastorno con elevada heredabilidad, entre un $70-80 \%$. Además, para comprender la neuropsicología del TDAH es necesario conocer los conceptos de como las funciones ejecutivas permiten llevar a cabo una conducta eficaz, creativa y socialmente aceptada, así como la memoria operativa que es la actualización y mantenimiento de la información durante un periodo corto de tiempo, así como los modelos neuropsicológicos que los fundamentan.

Por ello, la presente investigación tiene como objetivo identificar las metodologías trabajadas en estudiantes que presentan el Trastorno de Déficit de Atención e Hiperactividad y sus niveles de relación entre pares y el rendimiento en sus clases.

\section{MATERIALES Y MÉTODOS}

El presente estudio corresponde al enfoque cuantitativo, tipo básico, diseño no experimental, investigación documental descriptiva. El investigador examina, detalla y establece varios aspectos del fenómeno o materia de indagación. Uso de la sistematización de la revisión de investigaciones en revistas publicadas indexadas basadas en el análisis de las principales informaciones sobre el Trastorno por Déficit de Atención e Hiperactividad (TDAH). Se realizó una revisión bibliográfica que consistió en la búsqueda de diferentes estudios en las bases de datos Psyclnfo, Dianet, MedLine, Redalyc, Scielo y PubMed, Revistas Neurológicas, Revistas Sicológicas utilizando las palabras claves Trastorno por Déficit de Atención e Hiperactividad, TDAH, en español y en inglés Attention Deficit Hyperactivity Disorder. Los criterios de inclusión fueron: a) artículos con estudios experimentales b) artículos en español e inglés c) artículos más citados por la comunidad científica y d) artículos en base a las categorías y descripciones.

\section{RESULTADOS}

La cantidad de fuentes de información identificada en el presente estudio se presenta en la Tabla I.

Tabla I Cantidad de artículos identificados por base de datos

\begin{tabular}{ll}
\hline INVESTIGACIONES & TOTAL \\
\hline Redalyc & 6 \\
Scielo & 4 \\
Pubmeb.gov & 7
\end{tabular}




$\begin{array}{lr}\text { Science Direct } & 2 \\ \text { Dialnet } & \text { I } \\ \text { APA Psycnet } & 6 \\ \text { Revistas } & \text { de } \\ \text { Neurología } & 4 \\ \text { Revistas de Sicología } & 6\end{array}$

En el estudio, se seleccionados 18 artículos para realizar su análisis y se presentan en la Tabla 2.

Tabla 2 Análisis de los artículos seleccionados

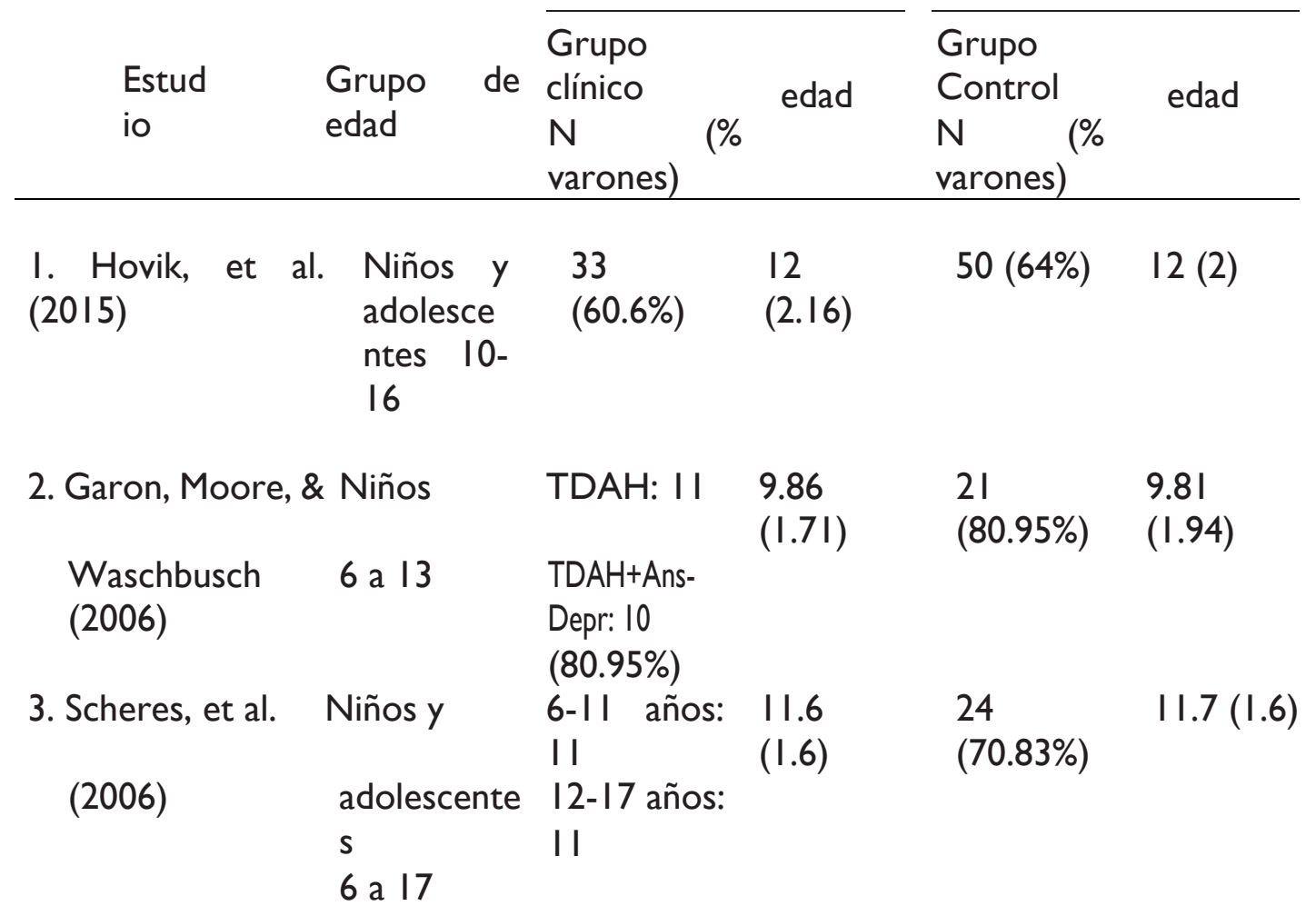
4. Toplak et al.Adolescent (2005) es (73.91\%)

5. Drechsler et al. Niños $23(91.3 \%)$ 24 $(95.83 \%)$ (2008) II a 13 6. Luman, Niños 23 Oosterlaan, 23
$(78.26 \%)$

$\begin{array}{lll}9.65 & 20(75 \%) & 9.43 \\ (I .4 I) & & (1.37)\end{array}$

Knol, \& Sergeant 7 a 12 (2008) 


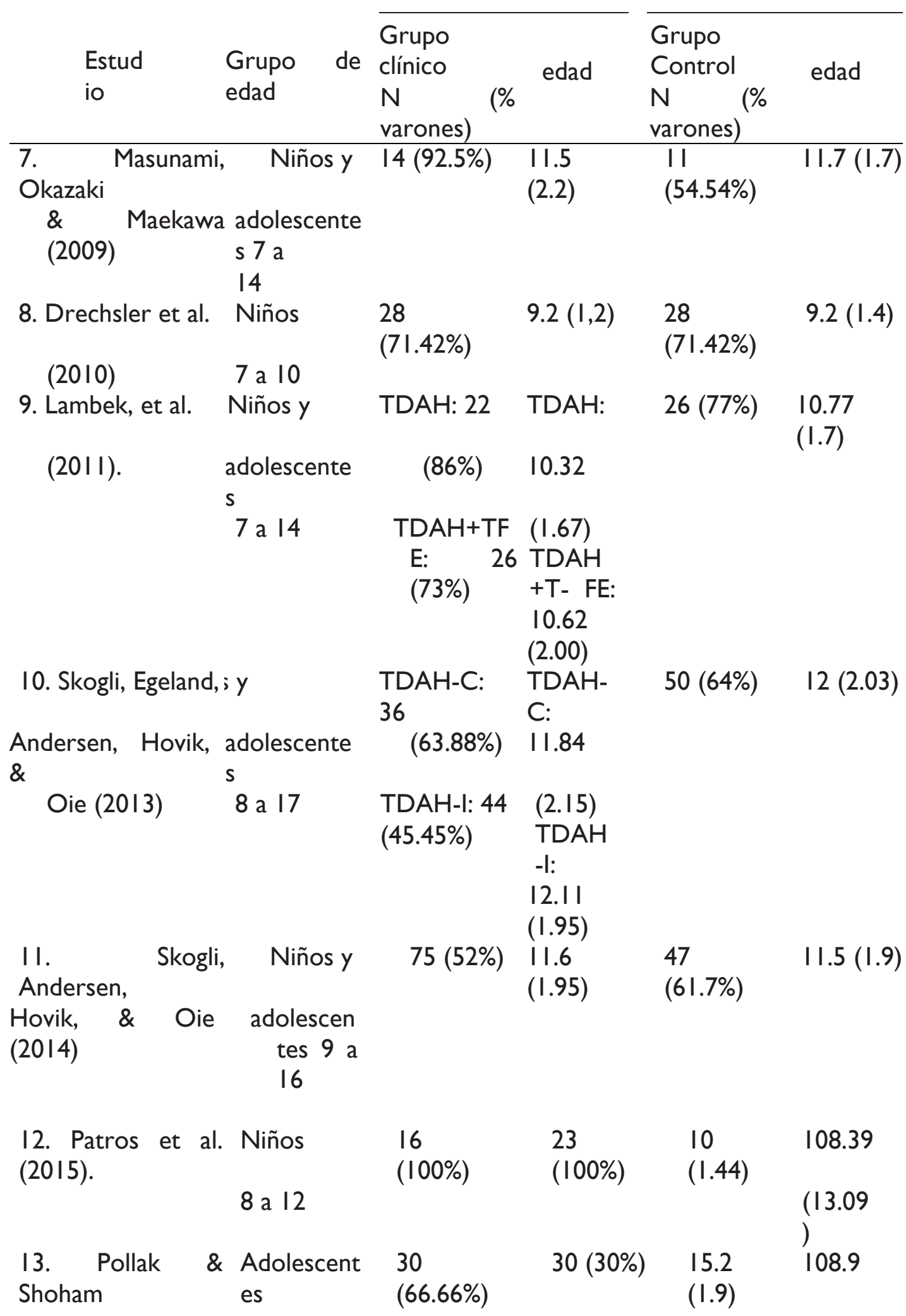




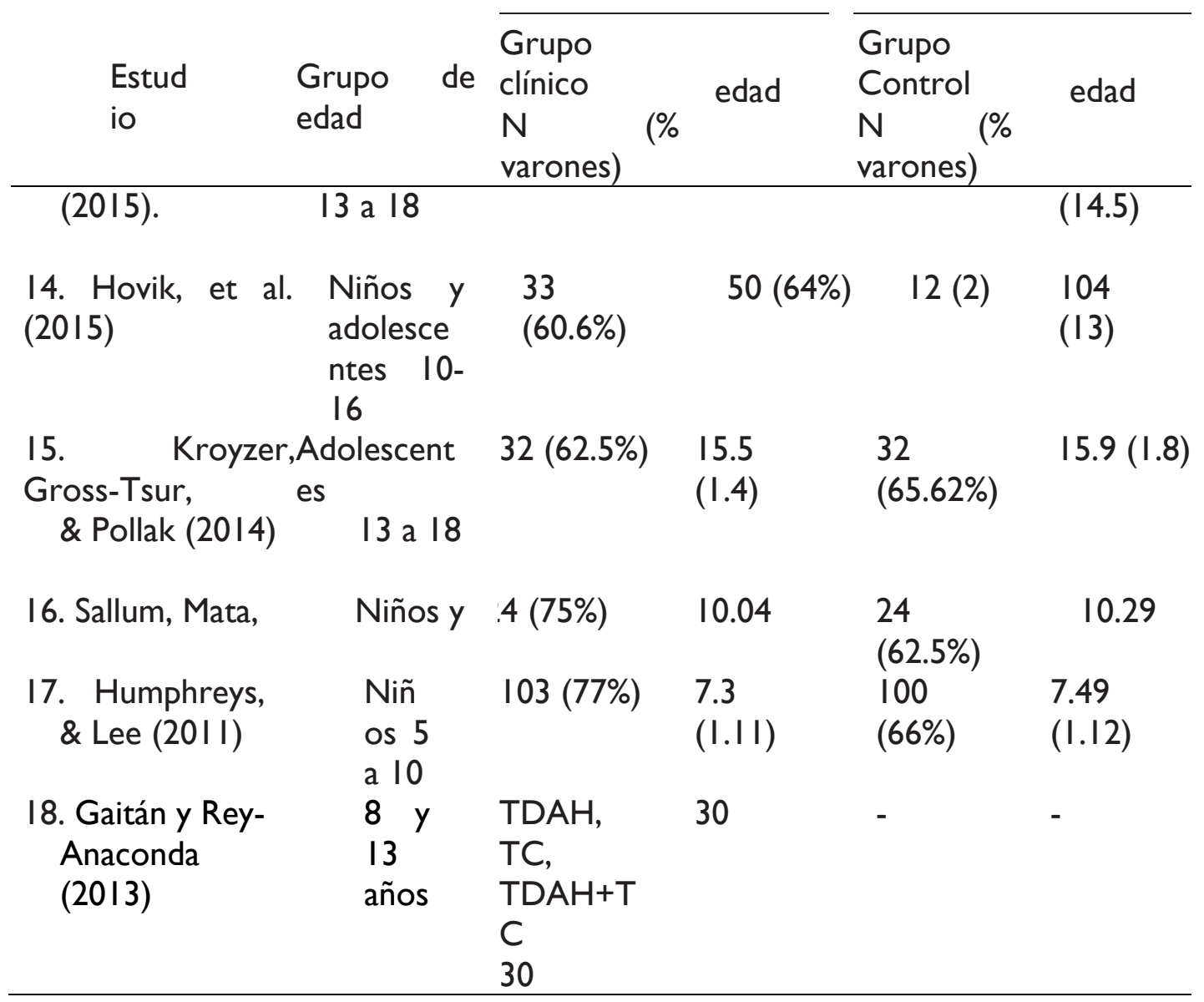

Los artículos incluyeron información sobre la cantidad, edad, sexo, la población. Las investigaciones mostraron que un el $45 \%$ se realizaron solo con niños hasta los 13 años de edad, el $40 \%$ se realizaron con niños y adolescentes, I5\% sólo con adolescentes entre las edades de 13 a 18 años. La totalidad de los estudios incluyeron información sobre el número de sujetos y los grupos de edad incluidos en la muestra. En las evaluaciones realizadas con el DSM-5 se encontró una mayor presencia del género masculino que femenino. Kroyzer, Gross-Tsur, y Pollak (2014) observaron que los adolescentes con TDAH eligen la opción menos probable con más frecuencia que los estudiantes que no presentan TDAH independientemente del subtipo, sexo y el nivel intelectual, pero presentan la misma velocidad a la hora de tomar decisiones. Pollak y Shoham (20I5) observaron que la toma de decisiones probabilística se asocia con el TDAH, los estudiantes realizaron una tarea con diferentes indicaciones, los estudiantes con TDAH, tuvieron dificultad a pesar que se les proporcionaron los resultados y probabilidades. Skogli et al. (20I3) en su estudio con estudiantes con TDAH de subtipo combinado (TDAH-C), subtipo inatento (TDAH-I), y un grupo control, no encontraron diferencias significativas entre ellas con respecto al rendimiento. Así mismo Méndez y Martínez (2010) observaron en su estudio diferencias en las conductas externalizantes, el 
rendimiento académico y la atención selectiva en estudiantes de 6 a II años con y sin TDAH, de estas variables la que más se ve afectada por el TDAH, es el rendimiento académico, más no la atención selectiva y la conducta externalizante, se encontró una mayor presencia del TDAH en varones que en mujeres.

Gaitán y Rey-Anaconda (2013) seleccionaron a un grupo de estudiantes que presentaban TDAH y/o TC y otro grupo de estudiantes que no presentaban ninguna dificultad a nivel académico y de comportamental entre las edades de 8 y 13 años arrojando como resultados una correlación entre inatención e hiperactividad e impulsividad de .37 en los niños y .45 en las niñas. Sallum, Mata, Miranda, y Malloy-Diniz (2013) observaron una puntuación similar en estudiantes con y sin TDAH en el desarrollo de la prueba, pero encontraron diferentes estrategias durante la prueba, al cambiar la elección del mazo, los estudiantes con TDAH tienen un desplazamiento mayor que los estudiantes que no tienen TDAH. Patros et al. (20I5) los estudiantes con TDAH respondieron de forma impulsiva a una tarea de cinco opciones que los niños sin TDAH sustentando que la impulsividad es más notoria en los estudiantes que presentan TDAH.

\section{DISCUSIÓN}

Moreno y Martínez (2010) estudiaron a niños de 6 a II años con y TDAH logró establecer diferencias en las conductas externalizantes, rendimiento académico y atención selectiva, encontrando que la única variable según el análisis factorial era el rendimiento académico. El trabajo de Bustillos y Servera (20I5) analiza la capacidad de los subtests, índices y perfiles basados en las escalas Wechsler para ayudar en el diagnóstico de TDAH, el $31 \%$ de niños diagnosticados con TDAH, presentaban una tendencia a puntuar igual o inferior a la muestra TDAH americana, por lo que muestra puede ser considerada claramente clínica. En cuanto a la comparación de la muestra clínica con la muestra control, ésta reportó diferencias significativas en 10 de los 15 subtests, encontrando un bajo rendimiento de los niños con TDAH en la Memoria de Trabajo, moderado/alto, en la Velocidad de Procesamiento y el $\mathrm{Cl}$ total. No se observaron diferencias en los índices de Comprensión Verbal o Razonamiento Perceptivo. Dekkers, Popma, Van Rentergem, Bexkens, y Huizenga, 2016; Groen et al., 20I3, evidenciaron que hay una heterogeneidad de las investigaciones sobre la TD en niños y adolescentes con TDAH, particularmente en la toma de decisiones, pero indican que los resultados, no son concluyentes y mantienen la controversia sobre el tema. Lo mismo se encontró en otras revisiones con niños y con población adulta. Para la American Psychiatric Association, 2013 con respecto al género, la totalidad de los estudios encontraron que mayormente niños y adolescentes evaluados de género masculino, presentaban una prevalencia del trastorno mayor que en el género femenino, sin embargo, no se observaron diferencias en cuanto al desempeño en la toma de decisiones. Drechsler et al., 2008; Drechsler et al., 2010; Garon et al., 2006; Luman et al., 2008; Humphreys y Lee, 20I I; Patros et al., 2015 observaron una relación a las características de los participantes, específicamente en niños entre las edades de 6 y 13 años, un desempeño inferior en referencia a los niños del grupo clínico. Pero en los 
estudios de Hovik et al., 20I5; Lambek et al., 20I I; Masunami et al., 2009; Scheres et al., 2006; Skogli et al., 20I4; Skogli et al., 2013 que trabajaron con muestras mixtas el $75 \%$ de los niños y adolescentes no encontraron diferencias en las medias de los desempeños de ambos grupos. Kroyzer et al., 20 I4; Pollak y Shoham, 20I5; Toplak et al., 2005 en la totalidad de los estudios que analizaron sólo encontraron evidencias de un desempeño inferior de los jóvenes con TDAH. Los resultados respecto a lo que sucede con los adolescentes son consistentes con los estudios de Bernal, Montaña, Acosta y Rojas (2015), que indican que, a partir de los 14 años, los avances en el funcionamiento cognitivo, no presentan una mejora gradual en el desempeño en la toma de decisiones, esto se debe a un aumento de la búsqueda de sensaciones y a la adopción de comportamientos de riesgo teniendo como tendencia el alejarse de la norma, afectando el proceso de TD (Reyna y Farley, 2006). Gaitán y Rey-Anaconda (2013) los resultados obtenidos en esta investigación confirman que existe una alta probabilidad que los niños con TDAH, TC y TDAH+TC presenten mayores déficits en el funcionamiento ejecutivo, en comparación con niños que no presentan ningún trastorno, estos podrían interferir significativamente a nivel cognitivo, emocional y de comportamiento en las diferentes actividades personales, familiares, sociales y académicas en las que se desenvuelven los niños. Por esta razón sería importante intervenir positivamente en el desarrollo de habilidades ejecutivas de estos niños para mejorar su calidad de vida. Cardona y Varela (2016) analizan el desempeño lector y determinando la relación de variables neuropsicológicas con la edad, el subtipo y el carácter de la institución en niños diagnosticados con TDAH y un grupo control. Al observar las características del desempeño lector, considerando la edad como variable independiente se observa que, conforme avance la edad de los participantes, alcanzan un mayor número de aciertos debido a sus procesos de maduración que son correlativos con la edad, en donde las destrezas lectoras aumentan también en función del grado escolar. Piñón, Carballido, Vásquez, Fernández, Gutiérrez y Spuch (2019) observaron que el rendimiento cognitivoejecutivo de una muestra clínica de niños y niñas diagnosticados con TDAH al comparándolo con un grupo normativo, los niños con diagnóstico presentan alteraciones en los subprocesos atencionales y en el funcionamiento ejecutivo. Los datos obtenidos no concuerdan con la hipótesis inicial en la que proponían que los niños con TDAH presentan alteraciones en los subprocesos atencionales, en el funcionamiento ejecutivo, pero sí encuentran algunas dificultades en atención selectiva e inhibición conductual del grupo clínico. Sin embargo, los datos sí son concordantes con la hipótesis de que existen diferencias entre los niños con TDAH y un grupo normativo, en donde se encontró un pobre desempeño en el grupo con TDAH en comparación con el grupo normativo. Una de las limitaciones del presente estudio es que a pesar de tener una gran gama de información hay muchas variantes que se deberían de investigar como es el aspecto familiar y social de los estudiantes diagnosticados con TDAH. 


\section{REFERENCIAS}

American Psychiatric Association (2013). DSM-5. Manual diagnóstico y estadístico de los trastornos mentales. Barcelona: Masson.

Barrios Omar, M., Ramírez-Dueñas, M., Chamorro, Y., \&Trejo, B., (2016). Características del trastorno por déficit de atención e hiperactividad en escolares mexicanos de acuerdo con la percepción de los padres, Suma Psicológica, 23(2), I0I-I08.

Bernal, P., Montaña, J., Acosta, R., \& Rojas, Y. (20I5). Performance of Children and Adolescents from a School of the City of Sogamoso on a Decision-Making Test. Open Journal of Pediatrics, 5, 339-347. doi: 10.4236/ojped.2015.5405I.

Bustillo, M., \& Servera M. (20I5). Análisis del patrón de rendimiento de una muestra de niños con TDAH en el WISC-IV. Revista de Psicología Clínica con Niños y Adolescentes, 2(2), I2I-I 28.

Cardona, M. \& Varela, V. (20I7). Desempeño lector en niños con diagnóstico de TDAH (Trastorno de Déficit de Atención con Hiperactividad). Psicogente, 20(37), 99II7.

Cardo, E., \& Servera, M. (2008). Trastorno por déficit de atención/ hiperactividad: estado de la cuestión y futuras líneas de investigación. Revista de Neurología, 46, 365-372.

Chávez, B. (2020). El diseño de una estrategia de evaluación de los aprendizajes de niños y niñas con características diversas: un aporte para transformar el aula. Revista Científica de Ciencias Sociales, 4(I), II4-I25. http://www.revistasocialium.com/index.php/es/article/view/52/html

Dekkers, T. J., Popma, A., Van Rentergem, J. A. A., Bexkens, A., \& Huizenga, H. M. (2016). Risky decision making in Attention-Deficit/Hyperactivity Disorder: A metaregression analysis. Clinical Psychology Review, 45, I-16. doi: 10.1016/j. cpr.2016.03.00I.

Drechsler, R., Rizzo, P., \& Steinhausen, H. C. (2008). Decision-making on an explicit risktaking task in preadolescents with attention-deficit/hyperactivity disorder. Journal of Neural Transmission, I I 5, 20 I-209. doi: 10.1007/s00702-007-08|4-5.

Drechsler, R., Rizzo, P., \& Steinhausen, H. C. (2010). Decision making with uncertain reinforcement in children with attention deficit/hyperactivity disorder (ADHD). Child Neuropsychology, 16, 145-16I. doi: 10.1080/09297040903190774.

Kroyzer, N., Gross-Tsur, V., \& Pollak, Y. (20I4). Risk taking in adolescents with attention defi cit hyperactivity disorder on a probabilistic choice task. The Journal of nervous and mental disease, 202, 247-252. doi: 10.1097/NMD.0000000000000I00.

Gaitan Chipatecua, A., \& Rey-Anaconda, C.A. (20I3). Diferencias en funciones ejecutivas en escolares normales, con trastorno por Déficit de Atención e Hiperactividad, trastorno del cálculo y Condición Comórbida. Avances en Psicología Latinoamericana, 3 I (I), 7I-85. 
Garon, N., Moore, C., \& Waschbusch, D. A. (2006). Decision making in children with ADHD only, ADHD-anxious/depressed, and control children using a child version of the lowa Gambling Task. Journal of Attention Disorders, 9, 607-619. doi: I0.1 I77/I08705470528450I.

Hovik, K. T., Plessen, K. J., Cavanna, A. E., Skogli, E. W., Andersen, P. N., \& Oie, M. (20I5). Cognition, Emotion and Behavior in Children with Tourette's Syndrome and Children with ADHD-Combined Subtype-A Two-Year Follow-Up Study.

Humphreys, K. L., \& Lee, S. S. (20I I). Risk taking and sensitivity to punishment in children with ADHD, ODD, ADHD+ODD, and controls. Journal of Psychopathology and Behavioral Assessment, 33, 299-307. doi: 10.1007/ s 10862-01 I-9237-6.

Jiménez-Torres, Manuel G., y López-Sánchez, Manuel, \& Guerrero Ramos, Daniel (2010). Relación entre Experiencia Óptima percibida y rendimiento Académico percibida y Rendimiento Académico según la presencia de TDHA. Revista Mexicana de Psicología, 27 (I), I5-23.

Luman, M., Oosterlaan, J., Knol, D. L., \& Sergeant, J. A. (2008). Decision-making in ADHD: Sensitive to frequency but blind to the magnitude of penalty? Journal of Child Psychology and Psychiatry, 49, 712-722. doi: 10.1III/j.14697610.2008.01910.x

Llanos, L., García, D., González, H., Puentes, P. (2019). Trastorno por déficit de atención e hiperactividad (TDAH) en niños escolarizados de 6 a 17 años.

http://scielo.isciii.es/scielo.php?script=sci_arttext\&pid=SI I 3976322019000300004

Lavigne, R., \& Romero, J. (2010). Modelo Teórico del Trastorno por Déficit de Atención con Hiperactividad I: Definición Operativa. Electronic Journal of Research in

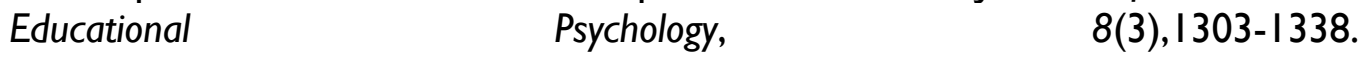
https://www.redalyc.org/articulo.oa?id=293 I/293 I 22000017

Lambek, R., Tannock, R., Dalsgaard, S., Trillingsgaard, A., Damm, D., \& Thomsen, P. H. J. (20II). Executive dysfunction in school-age children with ADHD. Journal of Attention Disorder, 15, 646-655. doi: 10.1 I77/10870547/0370935.

López-Martín, S., Albert, J., Fernández-Jaén, A., y Carretié, L. (2010). Neurociencia afectiva del TDAH: Datos existentes y direcciones futuras. Escritos de Psicología, 3, 17-29

Masunami, T., Okazaki, S., \& Maekawa, H. (2009). Decision-making patterns and sensitivity to reward and punishment in children with attention-defi cit hyperactivity disorder. International journal of psychophysiology, 72, 283-288. doi: 10.1016/j.ijpsycho.2009.01.007

Mediavilla-García, C. (2003). Neurobiología del trastorno de hiperactividad. Revista de Neurología, 36, 555-565.

Moreno Méndez, J., \& Martínez León, N. (2010). Conductas Externalizantes, Rendimiento Académico y Atención Selectiva en Niños con y sin Hiperactividad. Psicología. Avances de la disciplina, $4 \quad$ (I), 39-53. https://www.redalyc.org/articulo.oa?id=297224086004 
Patros, C. H., Alderson, R. M., Lea, S. E., Tarle, S. J., Kasper, L. J., \& Hudec, K. L. (20I5). Visuospatial working memory underlies choice-impulsivity in boys with attentiondeficit/hyperactivity disorder. Research in Developmental Disabilities, 38, I34-I 44. doi: 10.1016/j.ridd.2014.12.016

Pineda Santos, M. (2016). Trastorno por déficit de atención e hiperactividad en las escuelas de San Pedro Sula, 2016". Universidad Nacional Autónoma de Honduras en el Valle de Sula Escuela Universitaria de Ciencias de la Salud. http://www.bvs.hn/TMVS/pdf/TMVS34/pdf/TMVS34.pdf

Piñón, A., Carballido, E., Vasquez, E., Fernanadez, S., \& Gutierez, S. (2019). Rendimiento Neuropsicológico de niños y niñas con Trastorno por Déficit de Atención e Hiperactividad (TDAH). Panamerican Journal of Neuropsychology, I3(I), I I6-I 32. http://www.cnps.cl/index.php/cnps/article/view/360

Pollak, Y., \& Shoham, R. (2015). Feedback may harm: Role of feedback in probabilistic decision making of adolescents with ADHD. Journal of Abnormal Child Psychology, 43, 1233- 1242. doi: 10.1007/s 10802-015-0016-9.

Reyes, K. (2018). Maestro y lenguaje durante la educación básica: ¿formador de fracasos o productor de éxitos?. UNIVERSCIENCIA, I6(47), 43-48. http://revista.soyuo.mx/index.php/uc/article/view/85/87

Sallum, I., Mata, F., Miranda, D. M., \& Malloy-Diniz, L. F. (20I3). Staying and shifting patterns across IGT trials distinguish children with externalizing disorders from controls. Frontiers in psychology, 4, 899. doi: 10.3389/ fpsyg.2013.00899.

Sánchez-Encalada, L., \& Díaz, M. D. (2009). Detección de intervención a través del juego del TDAH. Revista de Psicología Iztacala, 12, I56-I70.

Scheres, A., Dijkstra, M., Ainslie, E., Balkan, J., Reynolds, B., Sonuga-Barke, E., \& Castellanos, F. X. (2006). Temporal and probabilistic discounting of rewards in children and adolescents: Effects of age and ADHD symptoms. Neuropsychologia, 44, 2092-2103. doi: 10.1016/j.neuropsychologia.2005.10.012.

Skogli, E. W., Egeland, J., Andersen, P. N., Hovik, K. T., \& Oie, M. (20I3). Few differences in hot and cold executive functions in children and adolescents with combined and inattentive subtypes of ADHD. Child Neuropsychology: A Journal on Normal and Abnormal Development in Childhood and Adolescence, 20, I-20. doi: 10.1080/09297049.2012.753998.

Skogli, E. W., Andersen, P. N., Hovik, K. T., \& Oie, M. (20I4). Development of Hot and Cold Executive Function in Boys and Girls With ADHD A 2-Year Longitudinal Study. Journal of Attention Disorders, I- I I. doi: I0. I I77/ I0870547/4524984.

Silva, Y., \& Velasquez, L. (2019). Artefactos pedagógicos: una experiencia inovadora. Centrosur. $3(1)$, 54-66. http://www.centrosureditorial.com/index.php/revista/article/view/25/49

Soutullo-Esperón C., \& Mardomingo-Sanz M. (2010). Manual de psiquiatría del niño y adolescente. Madrid: Editorial Médica Panamericana.

Reyna, V., \& Farley, F. (2006). Risk and Rationality in Adolescent Decisión Making: 
Implications for Theory Practice, and Public Policy. Psychological Science in the Public Interest, 7, I-44. doi: I0.1 I I I/j.I529-1006.2006.00026.x.

Tigrero, M. (2018). La formación integral de los estudiantes de básica media a través de la innovación desde el arte y la inteligencia emocional. Revista Perspectivas, (18), 35-46. https://revistas.uniminuto.edu/index.php/Pers/article/view/2286

Toplak, M. E., Jain, U., \& Tannock, R. (2005). Executive and motivational processes in adolescents with attention-defi - cit-hyperactivity disorder (ADHD). Behavioral and Brain Functions, I, 8. doi: 10.1 186/I744-908I-I-8.

Trujillo-Orrego, N., Ibáñez, A., y Pineda, D. E. (20/2). Validez del diagnóstico de trastorno por déficit de atención/hiperactividad: de lo fenomenológico a lo neurobiológico. Revista de Neurología, 54, 367-379.

Willcutt, E. G. (20I2). The prevalence of DSM-IV attention-defi-cit/hyperactivity disorder: a meta-analytic review. Neurotherapeutics, 9, 490-499. doi: I0.1007/s I33 II-0I2-0135-8.

Yasumura, A., Takimoto, Y., Nakazawa, E., \& Inagaki, M. (20I6). Decision Making in Children with Attention-Defi cit/ Hyperactivity Disorder. Open Journal of Pediatrics, 6, 158. doi: 10.4236/ojped.2016.62023 